\title{
Pursing of planar elastic pockets
}

\author{
Yann Bouremel ${ }^{\mathrm{a}, \mathrm{b}}$, Shivam Madaan ${ }^{\mathrm{c}}$, Richard Lee ${ }^{\mathrm{a}, \mathrm{c}}$, Ian Eames ${ }^{\mathrm{b}}$, Adam \\ Wojcik $^{\mathrm{b}}$, Peng Tee Khaw \\ ${ }^{a}$ National Institute for Health Research (NIHR) Biomedical Research Centre at \\ Moorfields Eye Hospital NHS Foundation Trust and UCL Institute of Ophthalmology, \\ London, UK \\ ${ }^{b}$ UCL Department of Mechanical Engineering, London, UK \\ ${ }^{c}$ UCL School of Pharmacy, London, UK
}

\section{Abstract}

The pursing of a simply- or doubly-connected planar elastic pocket by an applied pressure is analysed from the bending to the stretching regimes. The response is evaluated in terms of maximum deflection and profiles across a range of simply- and doubly-connected circular and square shapes. The study is conducted using experimental and numerical methods and supported by previous analytical results. The experimental method is based on an original 2D optical method that gives access to the pursing direction perpendicular to each image across the field of view. The equations for maximum pursing deflections are developed and compared for a range of thicknesses of silicone samples and shapes from the bending to the stretching regimes. In the case of doubly-connected shapes, dependence of maximum pursing deflection on clamped central circular and square areas or holes is quantified for both regimes. Good agreement is established between the three methods and the study also shows that the optical method may as well be successfully applied to problems of pursing of rubber pockets.

Keywords: elastic pocket, bending, stretching, hole, optical method, Finite 
Element Analysis

\section{Introduction}

An elastic pocket can be created by introducing a fluid between two elastic sheets fixed together along a common edge (Adkins and Rivlin, 1952). When the fluid exerts a pressure on the sheets, a purse is formed. Studies on inflating flat circular disks were initially carried out to understand elastic properties of gum rubber (Klingbeil and Shield, 1964; Hart-Smith and Crisp, 1967) with the eponymous model of strain-energy density from M. Mooney (Mooney, 1940) widely used to characterise incompressible and isotropic material such as rubber. His theory of large elastic deformation (Mooney, 1940) matches experimental tension data of soft rubber from compression to large stretching (50\%-400\% of original length). Pursed flat circular disks with clamped edge were therefore used to determine the strain energy density function of soft tissue (Wineman et al., 1979).

When considering a clamped pursed pocket, two regimes can be distinguished; when the deflection is smaller than the thickness of the initial flat pocket, the sheets bend and the equations of deflections are linear, while when the deflection is larger, the sheets are stretched. In the case of bending, the analytical solution of circular clamped pockets presents limited interest, and several groups have focussed on the analytical solutions for bending of rectangular or square plates with clamped edges (Timoshenko, 1910; Pistriakoff, 1910; Levy, 1942; Meleshko, 1997; Imrak and Gerdemeli, 2007a,b). Meleshko (1997) wrote a detailed account on the differences between each solution. More generally, a wide variety of studies have analysed elastic sheets that 
were pinned along an edge with the majority of studies focussed on circular or rectangular sheets that undergo large deformations (Dickey, 1967; Feng, 1976; Pujara and Lardner, 1978; Wineman, 1978; Roberts and Green, 1980; Weinitschke, 1988; Chaudhuri and DasGupta, 2014). More specifically, Yang and Feng (1970) studied the large deformations of hyper-elastic (MooneyRivlin) circular membranes, with stretching rising up to $600 \%$ (meridian stretching). Christensen and Feng (1986) extended this description to include deflection of neo-Hookean materials; comparisons with the numerical results of Yang and Feng (1970) showed that the approximate model is accurate up to $400 \%$ strain. One of the limitations of their model is that the stretch ratio can be considered as averaged, as their model assumed a uniform inflation over the surface of the disk. More recently, Hewitt et al. (2015) developed an elastohydrodynamic lubrification theory that characterise deflection of elastic sheets under different conditions. Lister et al. (2013) applied an optical method to measure deflection with high resolution along a line when peeling sheets are bent or stretched. The same method was applied by Pihler-Puzović et al. (2015) to measure the shape of a circular elastic pocket in the stretching regime as a method of calibration.

For the last approximately 20 years, a large numbers of studies have focussed on inflating axisymmetric balloons for technology applications, for example in the context of artery interactions during stent placements (Liang et al., 2005; Prendergast, 2003; Martin and Boyle, 2013), in electro-elastomers used in novel prosthetic blood pumps (Goulbourne et al., 2007, 2004), in novel endoscopy devices (Glozman et al., 2010), as urinary sphincters for patients suffering from severe stress incontinence (Hached et al., 2014), as pressure 
sensitive buttons, to strain cells in a controlled manner (Smith et al., 1998) and even as a tool to break into rental cars on holidays! (by levering doors open, so that grapples can be inserted). Moreover, inflatable membranes are also used in a number of space applications such as solar sails and arrays, scientific ballooning, thermal shields, pressurised habitats in space, actuators and telescope mirrors (Marker and Jenkins, 1997; Patil and DasGupta, 2013) as well as in the development of airbags, suspensions for cushioning and shock absorbers (Kumar and DasGupta, 2013). Inflatable membranes as elastomer based tunable devices (Song et al., 2012) have been used in the development of dielectrophoretical tunable optofluidic devices, leading to a number of applications such as beam steers, optical switches and single pixel displays (Xu et al., 2013). Laparoscopic gastric banding is an example of a doubly-connected inflatable structure. They are annular silicon membranes loaded with a saline solution that, under different pressure loads, limit the passageway of food through the stomach to reduce the nutritional intake of morbidly obese patients (Morino et al., 2003). Finally, the understanding of deformed elastic membranes is applicable to biological systems, such as fetal dura mater (Kriewall et al., 1983; Bylski et al., 1986), cell membranes to plant cell walls (Chaplain and Sleeman, 1990; Davies et al., 1998; Selby and Shannon, 2009), cell replication and motility to pathogenesis of disease (Karimi et al., 2014; Jenkins and Leonard, 2015).

Much of the research on the physics of clamped inflatable pockets has been conducted either looking at analytical solutions for small deformations such as in the work of Meleshko (1997) among others, or for large deformations of shapes such as Yang and Feng (1970) as explained previously. This paper is 
novel as it looks at the differences between those two regimes, while including not only simply but doubly-connected shapes (presence of a hole in the shape) using a three-fold approach: numerical and experimental approaches backed up by previous analytical results. It is believed by the authors that the experimental optical method is used for the first time to assess the pursing of silicone pockets. This original method gives a 3D deformation field from a single plane of measurements.

\section{Mathematical model}

The elastic pocket is modelled as a membrane of initial thickness $T$ that sustains a deflection $h$ due to an applied pressure $p$, as shown in figure 1 . To understand the implication of pressurising an elastic pocket, we apply a scaling analysis to identify the adjustment in the bending and stretching

regime. The bending regime is examined explicitly. The response is studied using Finite Element Method (FEM) which is validated against the results from the bending regime. It is worth noting that studying large inflations with the maximum deflection $H$ of the order of the pocket size is not within the scope of this paper.

\subsection{Results from the scaling analysis}

From the energy balance equation, Landau and Lifshitz (1975) derived:

$$
p=\frac{E T^{3}}{12\left(1-\sigma^{2}\right)} \nabla^{4} h-T\left(\frac{\partial^{2} \chi}{\partial y^{2}} \frac{\partial^{2} h}{\partial x^{2}}+\frac{\partial^{2} \chi}{\partial x^{2}} \frac{\partial^{2} h}{\partial y^{2}}-2 \frac{\partial^{2} \chi}{\partial x \partial y} \frac{\partial^{2} h}{\partial x \partial y}\right),
$$




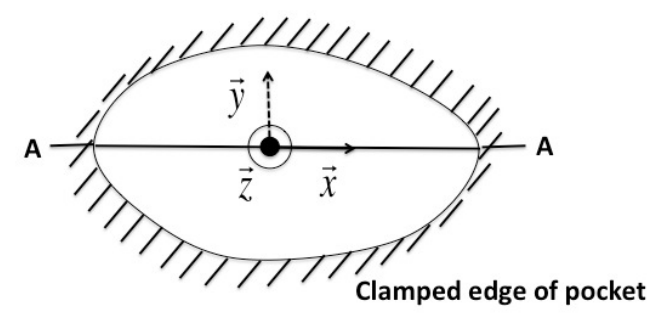

(a)

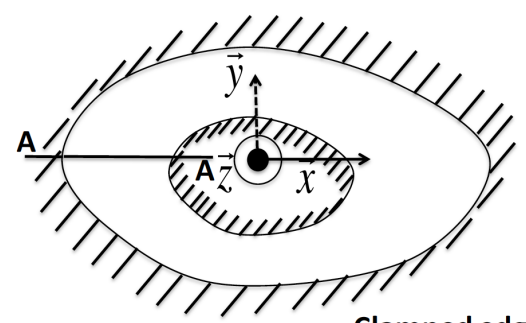

Clamped edges of pocket

(b)

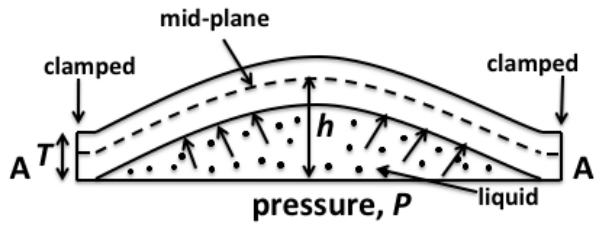

(c)

Figure 1: Schematic of plan-view of the problem showing the notation used and the boundary of (a) simply-connected and (b) doubly-connected pockets with the (c) side view. 
and

$$
\nabla^{4} \chi+E\left(\frac{\partial^{2} h}{\partial x^{2}} \frac{\partial^{2} h}{\partial y^{2}}-\left(\frac{\partial^{2} h}{\partial x \partial y}\right)^{2}\right)=0
$$

with $h$ being the deflection perpendicular to the $x-y$ plane; $E$, the Young's modulus; $\sigma$, the Poisson's ratio and $\chi$, a stress function. $\chi$ is defined in Landau and Lifshitz (1975) as $\zeta_{x x}=d^{2} \chi / d y^{2} ; \zeta_{x y}=-d^{2} \chi / d x d y$ and $\zeta_{y y}=$ $d^{2} \chi / d x^{2}$ with $\zeta_{i j}$, the stress tensor. Equations (1) and (2) are valid as long as the deflection, $h$, is smaller than the size of the pocket. The first term in the right hand-side of (1) represents the bending while the second term represents the stretching. A scaling analysis can be applied to identify the different regimes of pursing, by taking $H, L$ and $P$ as the characteristic scales for the pursed pocket deflection, size, and pressure. When $H / T \ll 1$, so that stretching is negligible, from (1):

$$
\frac{H}{T} \sim \frac{P L^{4}}{E T^{4}}
$$

The transition from bending to stretching occurs when $\frac{H}{T} \approx 1$ (Landau and Lifshitz, 1975). When stretching is important $(H / T \gg 1)$, then from $(1)$ :

$$
\frac{H}{T} \sim \frac{P L^{4}}{\chi T^{2}}
$$

and from (2):

$$
\chi \sim E H^{2}
$$

Eliminating $\chi$ gives an estimate of the pursed pocket gap to be:

$$
\frac{H}{T} \sim\left(\frac{P L^{4}}{E T^{4}}\right)^{1 / 3} .
$$

While the scaling analysis gives an estimate of the purse response in the two regimes, a more detailed analysis is required to determine the premultiplying coefficients. 


\subsection{Analytical results for bending}

When stretching $\chi$ is negligible, (1) reduces to the linear bending equation

$$
\frac{E T^{3}}{12\left(1-\sigma^{2}\right)} \nabla^{4} h=P
$$

which can be solved analytically in a number of cases. For a circular pocket, an axisymmetric solution to (7) can be derived (Chandrashekhara, 2001) and

$$
h(r)=\frac{3\left(1-\sigma^{2}\right)}{16 E T^{3}} P\left(R^{2}-r^{2}\right)^{2},
$$

with $R$ being the radius of the circular pocket and $r$, the radial coordinate. The maximum displacement $H$, which occurs in the centre of the pocket, is

$$
\frac{H}{T}=\frac{3\left(1-\sigma^{2}\right)}{16} \frac{P R^{4}}{E T^{4}} .
$$

If a circular region (radius $R_{1}<R$ ) is clamped in the centre of a circular pocket, (7) can be solved exactly with the following boundary conditions: $h=0$ and $\frac{\partial h}{\partial r}=0$ at $r=R_{1}$ and $r=R$. An exact solution is given at the end of the article from Amon and Widera (1969) and a simplified form is recalled in (10) with $\alpha=R 1 / R$ and $\beta=r / R$ :

$$
\begin{aligned}
& h(r)=\frac{3\left(1-\sigma^{2}\right) P R^{4}}{16 E T^{3}}\left(\left(\beta^{2}-1\right)^{2}+\right. \\
& \frac{(\alpha-1)(\alpha+1)^{3}\left(\alpha-\alpha \beta^{2}+A+\beta^{2}-B-1\right)}{2\left(\alpha^{2}+C-1\right)}+ \\
&\left.\frac{(\alpha-1)^{3}(\alpha+1)\left(\alpha-\alpha \beta^{2}+A-\beta^{2}+B+1\right)}{2\left(C-\alpha^{2}+1\right)}\right)
\end{aligned}
$$

with $A=2 \alpha \ln \beta, B=2 \beta^{2} \ln \beta$ and $C=2 \alpha \ln \alpha$. The deflection is reported for two limits of $\alpha$ : if $\alpha=0, h=\frac{3\left(1-\sigma^{2}\right) P \beta^{2} R^{4}}{16 E T^{3}}\left(\beta^{2}-2 \ln \beta-1\right)$ while when $\alpha=1, h=0$. 
For a rectangular pocket bounded by $|x| \leq a,|y| \leq b,(7)$ can be solved more easily in Cartesian coordinates:

$$
\frac{E T^{3}}{12\left(1-\sigma^{2}\right)}\left(\frac{\partial^{4}}{\partial x^{4}}+2 \frac{\partial^{4}}{\partial x^{2} \partial y^{2}}+\frac{\partial^{4}}{\partial y^{4}}\right) h=P .
$$

For a rectangular pocket (with all edges clamped), the solution derived by Koialovich (1902) and Boobnoff (1902) is:

$$
\begin{aligned}
h(x, y)= & \frac{12\left(1-\sigma^{2}\right)}{E T^{3}} \frac{P b^{4}}{24}\left[\left(1-\frac{y^{2}}{b^{2}}\right)^{2}+C_{0}+\sum_{k=1}^{\infty} A_{k} \sinh k \pi\right. \\
& \frac{(k \pi \operatorname{coth} k \pi+1) \cosh \frac{k \pi x}{b}-\frac{k \pi x}{b} \sinh \frac{k \pi x}{b} \cos \frac{k \pi y}{b}+}{k \pi+\cosh k \pi \sinh k \pi} \\
& \sum_{n=1}^{\infty} B_{n} \sinh n \pi \frac{(n \pi \operatorname{coth} n \pi+1) \cosh \frac{n \pi y}{a}-\frac{n \pi y}{a} \sinh \frac{n \pi y}{a}}{n \pi+\cosh n \pi \sinh n \pi} \\
& \left.\cos \frac{n \pi x}{a}\right],
\end{aligned}
$$

with $A_{k}, B_{n}$ and $C_{0}$ obtained from the systems of equations recalled in Meleshko (1997):

$$
\begin{gathered}
A_{k}=\frac{4}{\pi} \sum_{n=1}^{\infty} \frac{(-1)^{k+n+1} n^{3}}{\left(k^{2}+n^{2}\right)^{2}} \frac{\cosh (2 n \pi)-1}{\sinh (2 n \pi)+2 n \pi} B_{n}+48 \frac{(-1)^{k}}{k^{4} \pi^{4}}, \\
B_{n}=\frac{4}{\pi} \sum_{k=1}^{\infty} \frac{(-1)^{k+n+1} k^{3}}{\left(k^{2}+n^{2}\right)^{2}} \frac{\cosh (2 k \pi)-1}{\sinh (2 k \pi)+2 k \pi} A_{k}, \\
C_{0}=-\frac{2}{\pi} \sum_{k=1}^{\infty} \frac{(-1)^{k}}{k} \frac{\cosh (2 k \pi)-1}{\sinh (2 k \pi)+2 k \pi} A_{k} .
\end{gathered}
$$

In the case of a square $(b / a=1)$, the dimensionless maximum deflection, $H / T$, occurring at the centre has been derived by Boobnoff (1902) and is equal to:

$$
\frac{H}{T}=0.245\left(1-\sigma^{2}\right)\left(\frac{P a^{4}}{E T^{4}}\right)
$$

\subsection{Finite Element Analysis Model}

A finite element model of the three-dimensional elastic pocket was created and solved using Abaqus version 6.12-3. A linear model proves to be insuffi- 
cient for the stretching regime and therefore, the material was defined to be hyper-elastic, isotropic and homogeneous consistent with the experimental study. This model uses a strain energy density function, W (Mooney, 1940), which for incompressible elastic materials is described by:

$$
W=C_{1}\left(I_{1}-3\right)+C_{2}\left(I_{2}-3\right)
$$

where $I_{1}, I_{2}$ are the first and second invariants of the strain tensor and $C_{1}$, $C_{2}$ are material specific constants. The justification of using $W$ for modelling the silicone sheets is based on the relative low stretching of the pockets and the collapse of the experimental data onto a Mooney-Rivlin model. Different circular and square elastic pocket sizes and thicknesses were simulated for a given set of material constants $C_{1}$ and $C_{2}$. Typical domains consist in circle or square with $R=a=10 \mathrm{~mm}$ with a thickness of $0.1 \mathrm{~mm}$. During the simulation, the pressure is applied uniformly on the lower surface of the pocket. We used Newton's method to calculate solutions for pressure values covering 5 orders of magnitude in sequence. After each solution is found, the pressure is increased by step ranging from 0.0001 to 10 pascal depending on the pressure range of the simulation and the current solution is used as an initial guess for the next one. For a solution to converge, the largest correction of the solution for one increment should be less than $1 \%$ of the incremental change for the corresponding solution variable with a residual error of less than $0.5 \%$. The edges of the pockets are clamped. A mesh with 8-node linear brick (hex) elements was generated by extruding a 2D grid. The optimal number of elements is determined with the convergence of the maximum deflection $(H)$ and is usually around 50000 . 


\section{Experimental study}

Elastic pocket was created using a clear silicone sheet fixed between two clear acrylic plates - an upper plate and a base plate. The plate dimensions were $162 \mathrm{~mm}$ by $162 \mathrm{~mm}$. Geometries of different shapes were cut in the upper plate to fix the outer edge of the pocket. The pocket was pressurised by injecting water under the membrane through a hole drilled in the base plate, as shown in figure 2 .

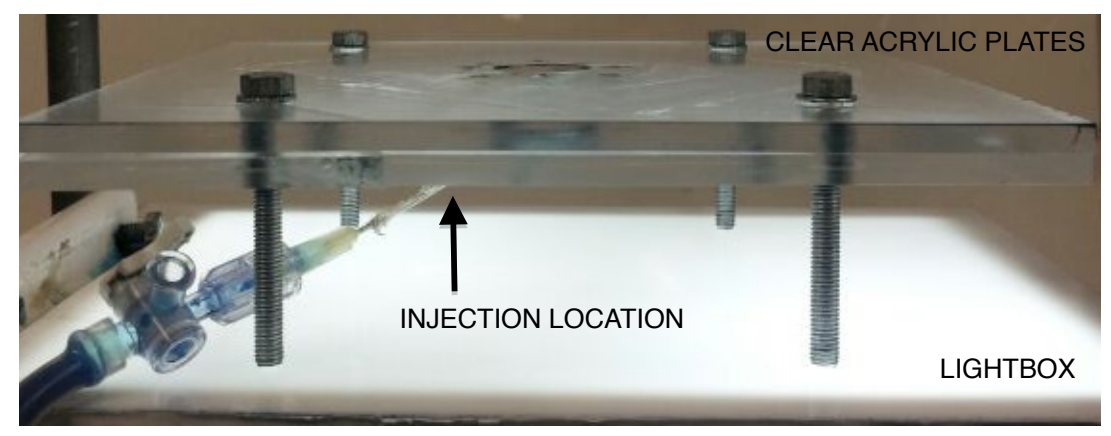

Figure 2: Photograph showing the lightbox and the upper and base plates screwed together holding sheet sample 2 . The needle is used to inject the dyed water through the base plate.

In order to apply a uniform hydrostatic pressure over the membrane, water columns of different heights were connected to the membrane. Since the height of the water column is significantly greater than $H$, the pressure applied over the membrane is uniform.

\subsection{Characterisation of sheet material properties}

Six silicone sheets (from Silex Ltd, Broxhead Trading Estate, Lindford, Bordon, Hampshire, GU35 OJX, UK) have been tested using the uniaxial tests 
to obtain the Mooney-Rivlin material constants $C_{1}$ and $C_{2}$ as well as the Young's Modulus E. The method of Vossoughi (1995) was used to obtain $C_{1}$ and $C_{2}$ from uniaxial tests by determining parameters $X$ and $Y$ :

$$
X=\frac{1-\lambda^{-3}}{\lambda-\lambda^{-2}}, \quad Y=\frac{\zeta}{2\left(\lambda-\lambda^{-2}\right)},
$$

as a function of the axial stretching ratio $(\lambda)$ and stress $(\zeta)$. Vossoughi (1995) showed that $C_{1}, C_{2}[$ from (15)] and $E$ can be determined from:

$$
Y=C_{1}+C_{2} X, \quad E=6\left(C_{1}+C_{2}\right)
$$

$C_{1}$ and $C_{2}$ are determined by the method of least squares.

\subsection{Characterisation of sheet deformation}

\subsubsection{Technique}

An optical method was used to determine the displacement of the elastic sheet to an applied pressure using a technique familiar in experimental fluid mechanics (e.g. Cenedese and Dalziel, 1998). This technique exploits the translucent (and almost transparent) nature of the silicone sheets. The sheet deformation was determined using a dilute methylene blue solution (a concentration of $33 \mathrm{mg} / \mathrm{L})$. The silicone sheet was clamped between two clear acrylic plates and lit with a diffusive light source. An Allied Vision technology camera of $1280 \times 960$ pixels was mounted with a $16 \mathrm{~mm}$ lens located above and perpendicular to the membrane to record the optical images (Fig. $3)$. 


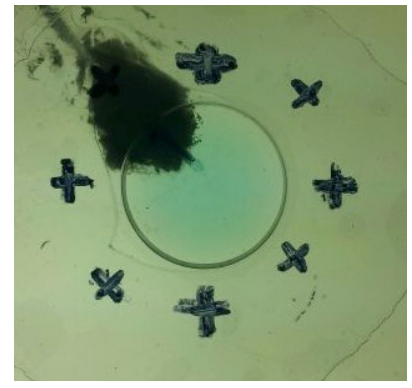

(a)

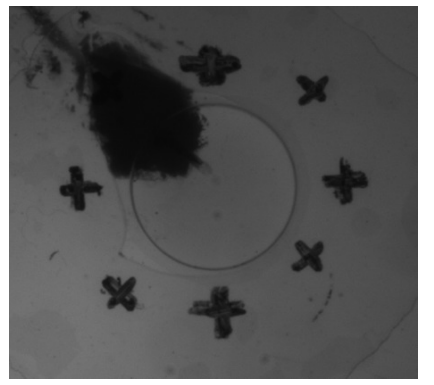

(b)

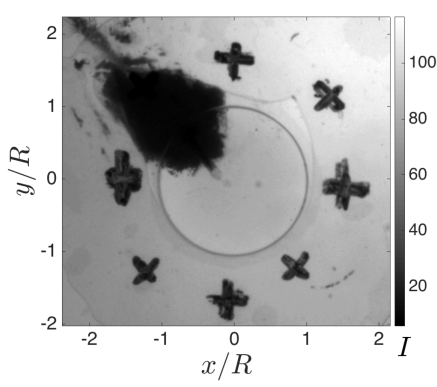

(c)

Figure 3: Typical example of the pocket with sheet sample 2 pursed with a non-dimensional pressure of 774 showing (a) a colour photograph of the pocket. In (b, c), the black-andwhite image recorded by the camera and post-processed are shown.

The governing principle of the optical technique was to correlate the height of the membrane to the attenuation of light intensity caused by dyed water using the calibration curve explained in section 3.2.2. The intensity of the methylene blue solution recorded by the 8-bit pixel camera is translated to a $0-255$ greyscale range, with 255 being no dye and 0 a very high amount of liquid.

\subsubsection{Calibration}

The optical method was calibrated against thin layers of dyed water that were created by successively adding $0.5 \mathrm{~mL}$ of dyed water to a measuring cylinder from a height of $0.82 \mathrm{~mm}$ up to $19.8 \mathrm{~mm}$. Figure 4 shows the variation of the fluid layer height with the ratio of intensity in greyscale of the current image compared to the reference image. The heights obtained from the optical method are on average accurate within $3.5 \%$. 


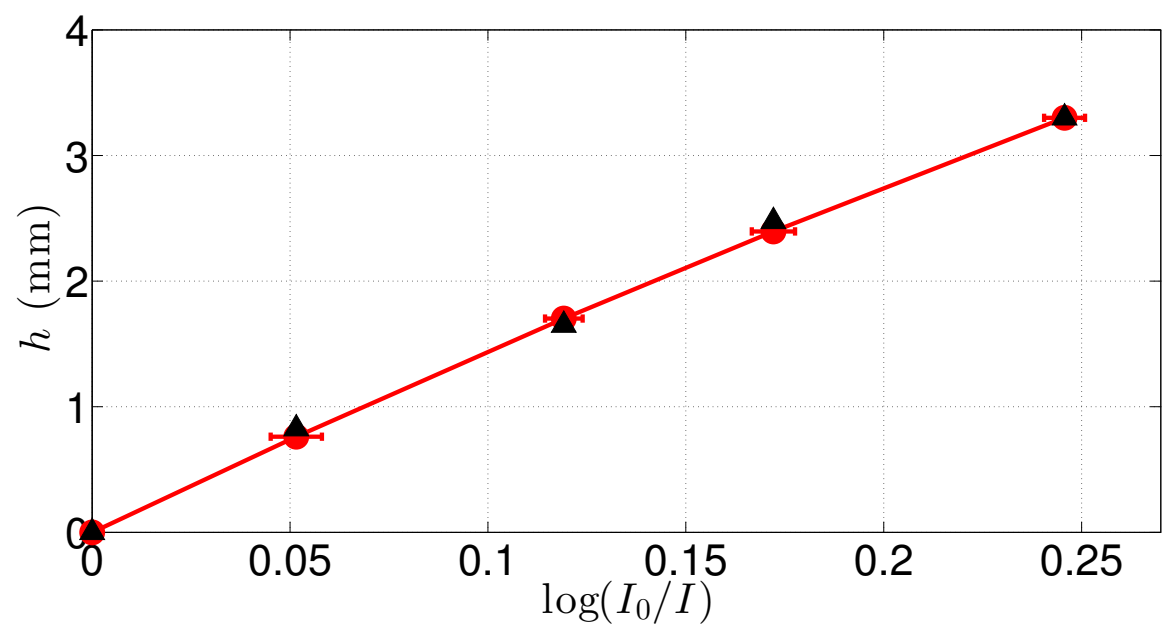

Figure 4: Calibration test showing the correlation between light attenuation determined optically $(\bullet)$ and layer thickness determined from a direct measurement $(\mathbf{\Lambda})$. It demonstrates (a) $\mathrm{h} \propto \log \left(I_{0} / I\right)$ and (b) the error is small (3.5\%) and very reproducible.

\section{Results}

\subsection{Material characterisation}

The six silicone sheets mentioned earlier with thicknesses ranging from 0.32 $\mathrm{mm}$ to $3.25 \mathrm{~mm}$ were characterised (see Table 1) using an electromagnetic linear actuator (Bose Electroforce 3220 Series III).

The maximum load applied is $225 \mathrm{~N}$ with a displacement of $6.5 \mathrm{~mm}$ and a resolution of $1 \mathrm{~nm}$. Each uniaxial tensile test was performed at a rate of $0.1 \mathrm{~mm} / \mathrm{s}$. This range of displacements was large enough to determine the Mooney-Rivlin parameters from the uniaxial tests.

Each silicone sample was cut into a bone-shape with the middle part being $4 \mathrm{~mm}$ wide and $15 \mathrm{~mm}$ long. Figure 5 shows a typical $X-Y$ variation for silicone sheet samples (in this case, samples 4,5 and 6). The values of 


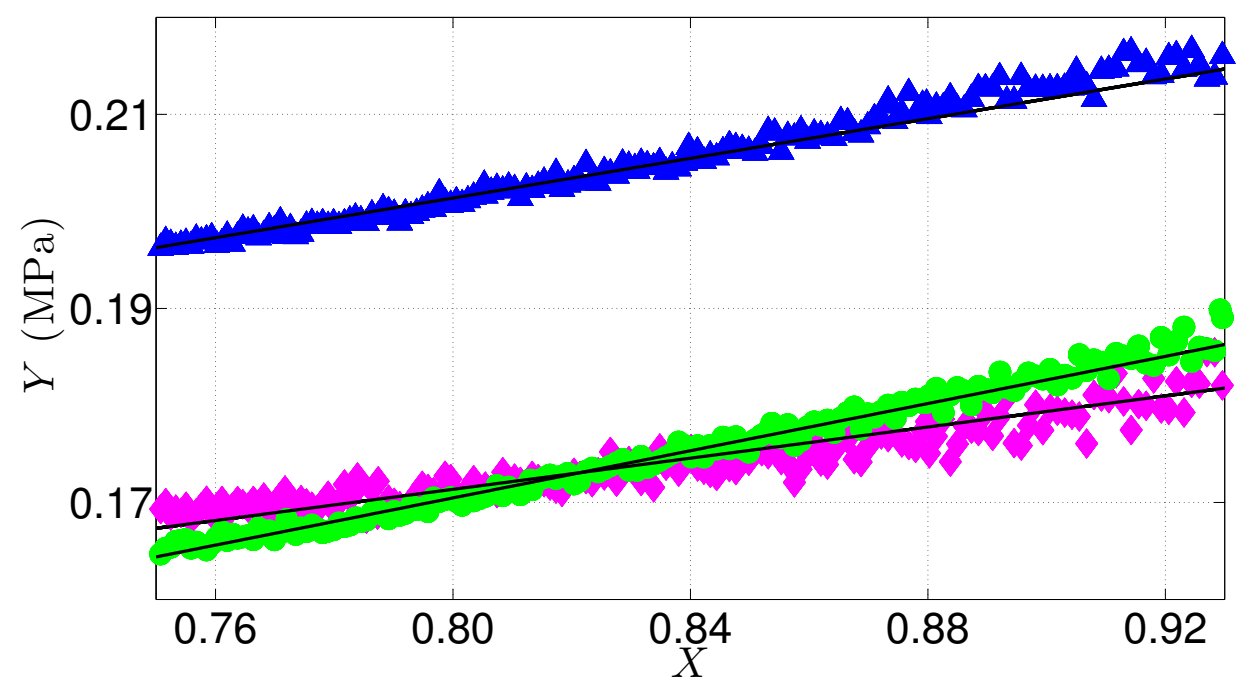

Figure 5: Typical $X-Y$ scatter plots for silicone samples 4, 5 and 6 (see Table 1). The Mooney-Rivlin parameters determined from the lines of best fit are also plotted.

$C_{1}$ and $C_{2}$ from each silicone sample were averaged from three tests.

\subsection{Simply-connected pockets}

\subsubsection{Field deformation}

This section aims at characterising the bending/stretching regime in terms of maximum deflections and profiles of different simply-connected shapes such as circles and squares. Typical elevated and plan-views of the deflection obtained experimentally using the optical method from the silicone sample 2 fitted in a circle of radius $10 \mathrm{~mm}$ are plotted in figure 6 (a)-(b), while in figure 6 (c)-(d), the deflection for the same geometry and sample are obtained numerically using Finite Element Analysis with each figure showing approxi- 


\begin{tabular}{|c|c|c|c|c|c|c|}
\hline $\begin{array}{l}\text { Silicone } \\
\text { sheet } \\
\text { sample }\end{array}$ & $\begin{array}{c}\text { Thickness } \\
T \\
(\mathrm{~mm})\end{array}$ & $\begin{array}{c}C_{1} \\
(\mathrm{kPa})\end{array}$ & $(\mathrm{kPa})$ & $\begin{array}{c}\text { Young's Modulus } \\
E \\
(\mathrm{MPa})\end{array}$ & $\begin{array}{l}\text { Length } \\
R \text { or } a \\
(\mathrm{~mm})\end{array}$ & Symbol \\
\hline 1 & 0.32 & 259 & 179 & 2.628 & 20 & $\nabla$ \\
\hline 2 & 0.5 & 160 & 47 & 1.241 & 10 & $\triangleright$ \\
\hline 2 & 0.5 & 160 & 47 & 1.241 & 20 & $>$ \\
\hline 3 & 1.6 & 160 & 47 & 1.241 & 10 & $\square$ \\
\hline 3 & 1.6 & 160 & 47 & 1.241 & 20 & $\square$ \\
\hline 4 & 1.72 & 122 & 64 & 1.112 & 10 & $\diamond$ \\
\hline 4 & 1.72 & 122 & 64 & 1.112 & 20 & $\vartheta$ \\
\hline 5 & 3.2 & 160 & 47 & 1.241 & 20 & $\Delta$ \\
\hline 6 & 3.25 & 48 & 133 & 1.083 & 20 & - \\
\hline
\end{tabular}

Table 1: Table summarising silicone samples used in the experimental study: the sheet thickness $(T)$, Mooney-Rivlin parameters $C_{1}$ and $C_{2}$, Young's Modulus $(E)$, length $(R$ or a), and symbols are listed. 


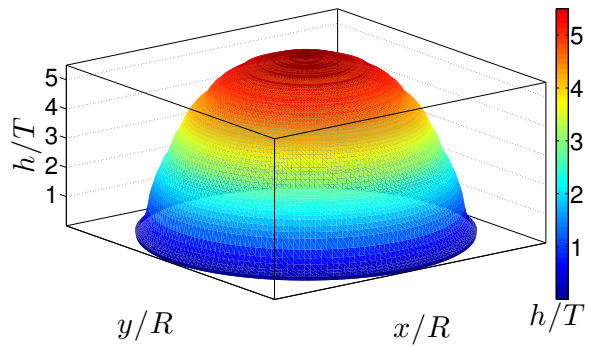

(a)

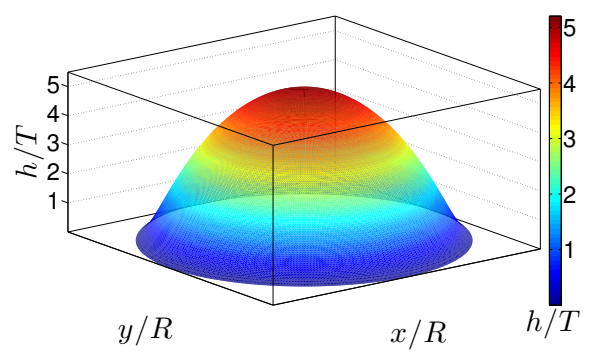

(c)

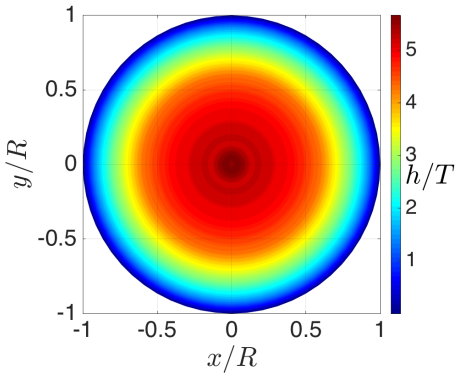

(b)

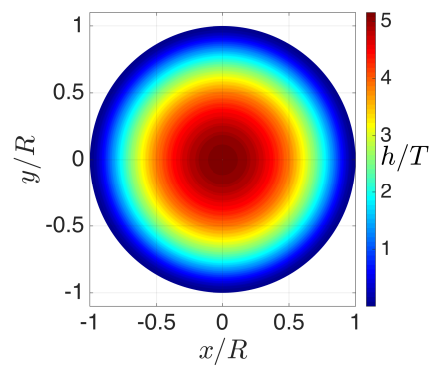

(d)

Figure 6: Elevated and plan-view contour plots of the reconstructed deformation of a circular silicone pocket of sheet sample 2 at a dimensionless pressure $P R^{4} / E T^{4}=580$ obtained (a-b) experimentally and (c-d) numerically using a finite element simulation (for $R=10 \mathrm{~mm})$.

mately identical maximum deflection. The experimental data are azimuthally averaged from the lower half of each image as the upper part of the field of view is obstructed by the injection point. The experimental elevated view is slightly rounder than the numerical one as confirmed by the plan-views. The clamping of the sheet may add extra compression on it leading to the slight raising of the silicone pocket, hence a slightly more stretched 3D view of the circular pocket. Identical figures are obtained for a square pocket of side $2 a=40 \mathrm{~mm}$ in figure 7 (a-d) for a dimensionless pressure $P a^{4} / E T^{4}=138$. 
In figure $7(\mathrm{a}, \mathrm{b})$, the experimental data have been smoothed. It is interesting to check if the contribution from the hydrostatic pressure to the overall relatively low applied pressure may be neglected. The dimensionless hydrostatic pressure difference between the centre and the edge can be calculated as $\rho g H a^{4} / E T^{4} \approx 0.94$ and considered negligible compared to the dimensionless pressure applied of 138 . The deflection is within the stretching regime. The difference in maximum deflections between the experimental and numerical pursed square pockets is within 10\%. However, the deformation occurring in the central part of the experimental pocket in figure 7 (a) appears flatter compared to the finite element results in figure 7 (c). However, when looking at the plan-view of figure 7 (b), it can be seen that the flattening occurs at the very top over a relatively small area of the entire pocket. It is therefore quite a minimum discrepancy and is probably due to the experimental technique and to a lesser degree the necessary smoothing of the experimental data, an additional step that is not required for the numerical results. The profiles are discussed in more detail in the following section.

\subsubsection{Profiles}

In figure 8, the cross-section profiles of simply-connected circular and square shapes for $r / R$ and $x / a$ from 0 to 1 obtained analytically, numerically and experimentally are plotted in the case of bending and stretching regimes. The simulations are obtained at respectively $P R^{4} / E T^{4}=1.4,58,174$ and 580 in figure 8 (a) for sheet sample 2 for a radius of $10 \mathrm{~mm}$ and $\mathrm{Pa}^{4} / E T^{4}=8.6,14$, 42 and 138 in figure 8 (b) for sheet sample 3 for $a=20 \mathrm{~mm}$ and plotted using solid lines. The equivalent bending analytical solutions (8) and (12) obtained 


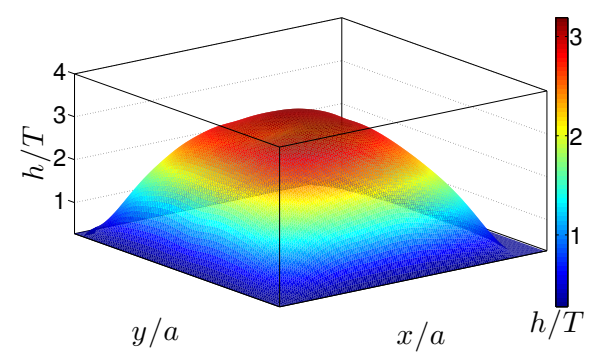

(a)

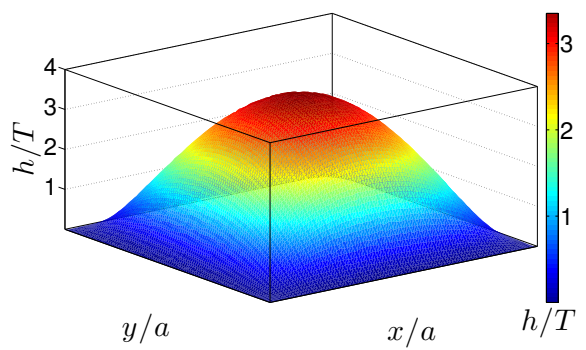

(c)

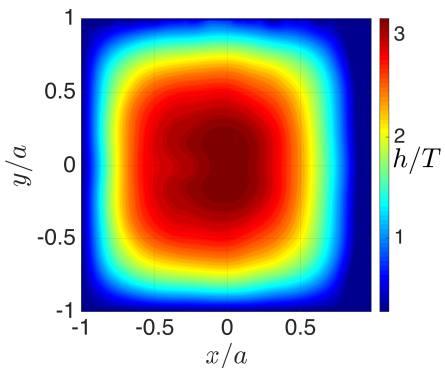

(b)

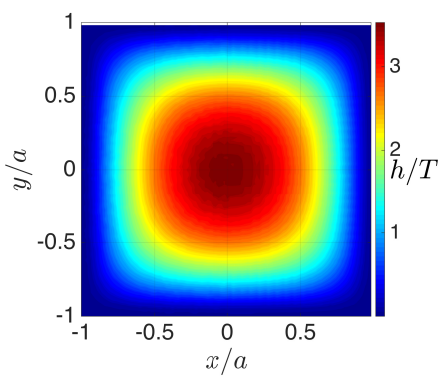

(d)

Figure 7: Elevated and plan-view contour plots of the reconstructed deformation of a square silicone pocket for sheet sample 3 at a dimensionless pressure $P a^{4} / E T^{4}=138$ obtained (a-b) experimentally and (c-d) numerically using a finite element simulation (for $a=20 \mathrm{~mm})$. 
for the circular and square shapes for $P R^{4} / E T^{4}=1.4$ and $P a^{4} / E T^{4}=8.6$, respectively, are plotted with a dashed line. It can be seen that the bending profile has a smaller width compared to the stretching profile in both circular and square shapes due to the fact that the membrane of the pocket is simply being displaced upwards under the applied pressure $p$ without being stretched when bent. However, the four experimental profiles obtained for the bending regime at $P R^{4} / E T^{4}=1.4(\bullet)$ and $P a^{4} / E T^{4}=8.6(\boldsymbol{\Delta})$ for the circular and square shapes respectively, and in the stretching regime at $580(\triangleright)$ and 138

(ם) for the circle and square shapes have a significant larger width compared to their theoretical and numerical equivalent profiles shown using the same colour. This could be due to the compression of the sheet when clamped to the acrylic plate that raises the sheet inside the circular or square shape, leading to artificially slightly larger deflections. However, the narrower width profile between the bending and stretching regime is clearly verified for all four experimental curves.

\subsubsection{Maximum displacements}

Figure 9 shows the dimensionless maximum deflection $(H / T)$ of circular and square elastic pockets over six orders of magnitude of $P R^{4} / E T^{4}$ and $P a^{4} / E T^{4}$. In figure 9 (a) and (b), the finite element simulations match respectively the bending solution for the circle (9) and for the square (14) plotted with red curves up to the maximum deflection of $H / T \approx 1$. Above $H / T \approx 1$, the silicone pocket undergoes stretching and the data obtained numerically can be fitted, in the case of the circular shape, with the following 


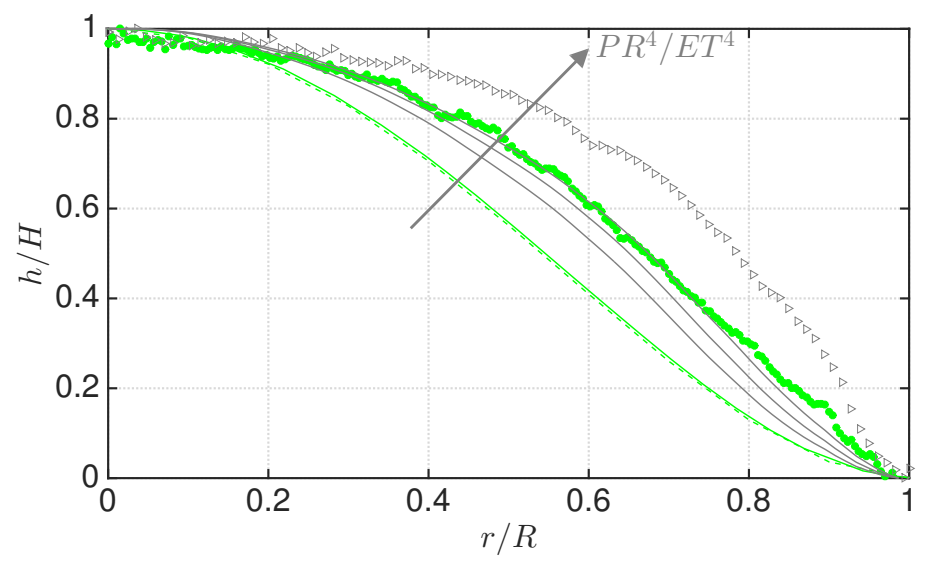

(a)

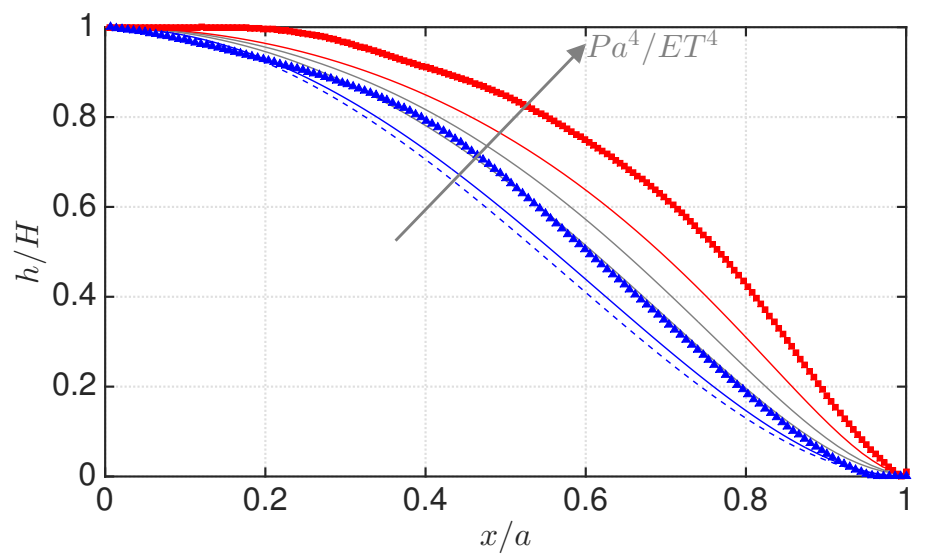

(b)

Figure 8: Profiles of the sheet deflection along a line of symmetry for (a) circular and (b) square pockets, as a function of dimensionless pressure $P R^{4} / E T^{4}$ and $P a^{4} / E T^{4}$ obtained analytically using (a) (8) and (b) (12) (dashed line), numerically using a finite element simulation ranging from $P R^{4} / E T^{4}=1.4-580$ and $P a^{4} / E T^{4}=8.6-138$ (solid line) and experimentally (see Table 1 ). The experimental results are plotted for the bending regime at $P R^{4} / E T^{4}=1.4$ (a) and $P a^{4} / E T^{4}=8.6$ (b) with the corresponding analytical and numerical results shown with the same colours. The experimental results are also plotted for the stretching regime at $P R^{4} / E T^{4}=580$ (a) and $P a^{4} / E T^{4}=138$ (b) with the numerical lines using identical colours. 
equation:

$$
\frac{H}{T} \approx \gamma\left(\frac{P R^{4}}{E T^{4}}\right)^{\frac{1}{3}}
$$

$R$ is replaced by $a$ in the case of a square shape. In the case of a circle, $\gamma$ is equal to 0.60. The coefficient $\gamma$ obtained with the current approach can be compared with previous findings. Gent and Lewandowski (1987) obtained a value of 0.595 using Hencky (1915) series expansion technique. Chien (1948); Dickey (1967); Kao and Perrone (1971) and Kelkar et al. (1985) found out the same value of 0.595 with different computing techniques while Christensen and Feng (1986) obtained 0.572. Our current value of 0.60 is within $1 \%$ of the value obtained by Hencky (1915). In the case of the square shape, $\gamma$ is equal to 0.70 . In the literature, Jones (1974) found numerically a value of 0.71 which is within $1.5 \%$ of our current values. The dimensionless maximum deflection, $H / T$, measured optically is also plotted. The discrepancies in greyscale on each measurement are reported with errorbars that are smaller than the symbol used to represent the averaged maximum deflections, $H / T$. Each case was repeated two times. It can be noticed that the data obtained from the optical method follows (9) for the circle and (14) for the square, validating the experimental method. The numerical results have been obtained from a serie of twelve simulations (six for the circle and six for the square) with $C_{1}=160 \mathrm{kPa}$ and $C_{2}=47 \mathrm{kPa}$; the thickness of the pocket, $T$, was fixed to $0.1 \mathrm{~mm}$, the length, $R$ or $a$, to $10 \mathrm{~mm}$ and the pressure, $P$, was increased up to $1000 \mathrm{~Pa}$ by step ranging between 0.0001 and $10 \mathrm{~Pa}$. The experimental, analytical and computational results agree quite well especially in the stretching regime except in the case of the sheet 2 for a circle radius of $10 \mathrm{~mm}$ with a maximum error of $40 \%$ on the height. It can be noticed that 
the experimental data do not exactly match, as well, the analytical results plotted with a red line in the bending regime; this may be due to the compression of the sheets by the acrylic plates when tightened to create a seal. The clamping can cause the sheet to be raised, which would more greatly affects the dynamics of pursing for smaller deflections in the bending regime compared to larger deflections in the stretching regime. 


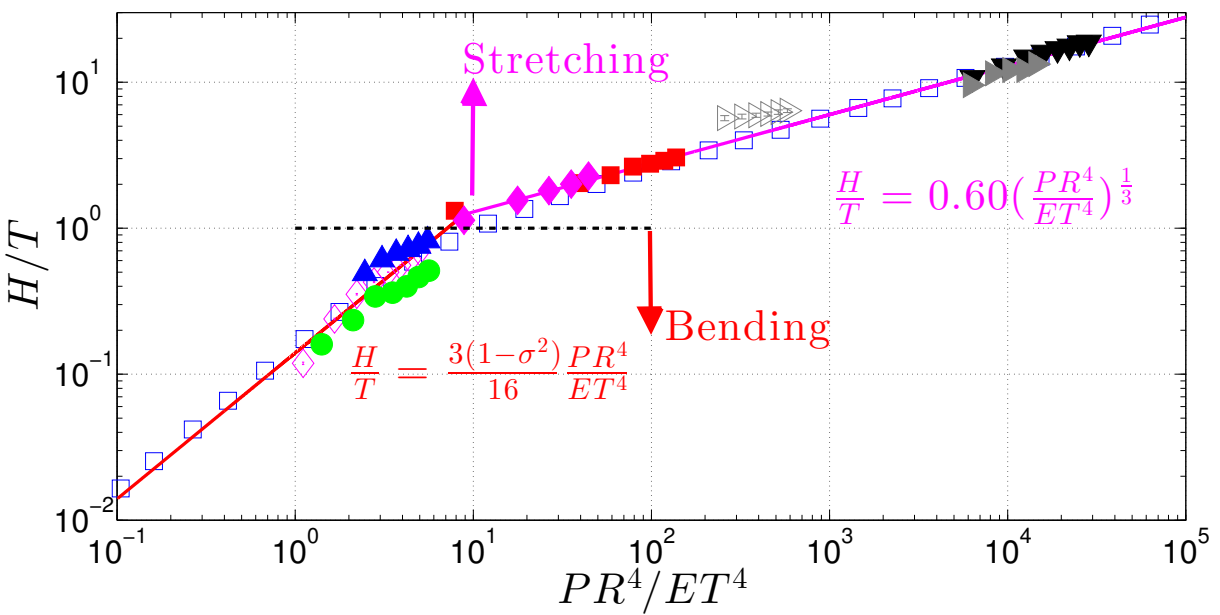

(a)

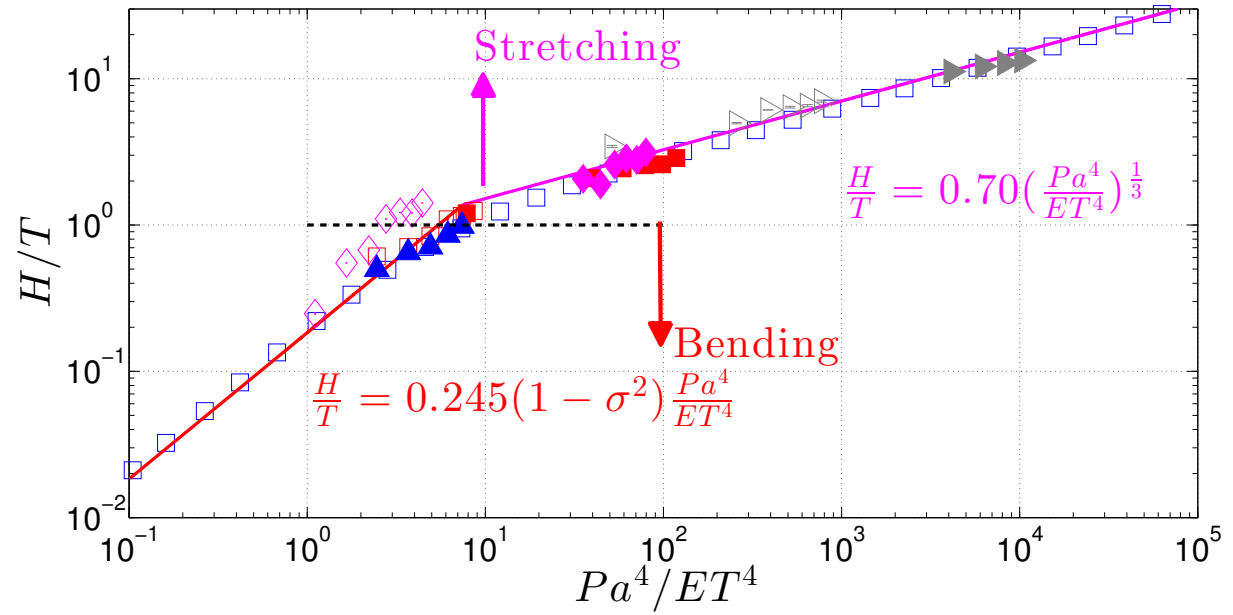

(b)

Figure 9: Variation of the dimensionless maximum deflection $(H / T)$ with the dimensionless pressure $\left(P R^{4} / E T^{4}\right.$ or $\left.P a^{4} / E T^{4}\right)$ for two different simply-connected shapes obtained numerically using finite element $(\square)$ and experimentally for a range of silicone samples (see Table 1). The red curve is the analytical result for the bending regime and the magenta curve is obtained numerically for the stretching regime: (a): circles; (b) squares. It is worth nothing that each experimental data point is reported with errorbars. 


\subsection{Doubly-connected pockets}

\subsubsection{Field deformation}

Doubly-connected pockets are characterised by the ratio of clamped area over total pocket area (both areas having similar shapes) or $\alpha=R_{1} / R$ in the case of a doubly-connected circle and $\alpha=a_{1} / a$ in the case of a doublyconnected square with $R_{1}$ and $2 a_{1}$ being the clamped radius and clamped side, respectively.

Numerical elevated and plan-views of doubly-connected circular and square pockets with a circular and square central clamped regions are obtained at similar dimensionless pressure $P R^{4} / E T^{4}=580$ and $P a^{4} / E T^{4}=138$ to figures 6 and 7 and shown in figure $10(\mathrm{a}-\mathrm{d})$. It can be seen that in figure 6 the maximum height $H$ goes from $5.2 T$ for a simply-connected circular shape to slightly below $2 T$ for a doubly-connected circular shape in figure 10 (a-b) with $\alpha=0.2$ and $P R^{4} / E T^{4}=580$, hence a reduction of around $60 \%$. Similarly, at $\mathrm{Pa}^{4} / E T^{4}=138$, the maximum deflection goes from $3.2 \mathrm{~T}$ for a simply-connected square as shown in figure 7 to $1.3 T$ for a doubly-connected square with $\alpha=0.2$ in figure $10(\mathrm{c}-\mathrm{d})$, hence a reduction of around $60 \%$. While the simply-connected shapes were clearly in the stretching regime, the presence of a clamped central region or hole results in the equivalent doublyconnected shapes to be closer to the bending regime, yet still in the stretching regime. This will be discussed in more detail in figures 12 and 13 . 


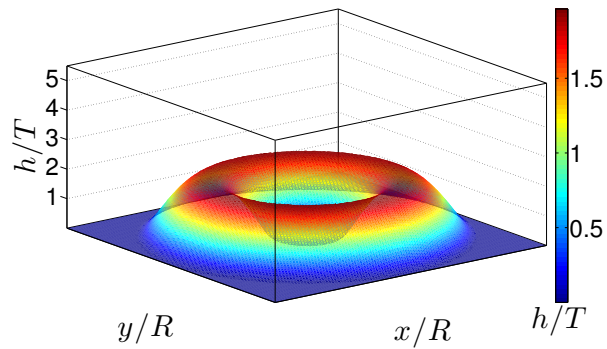

(a)

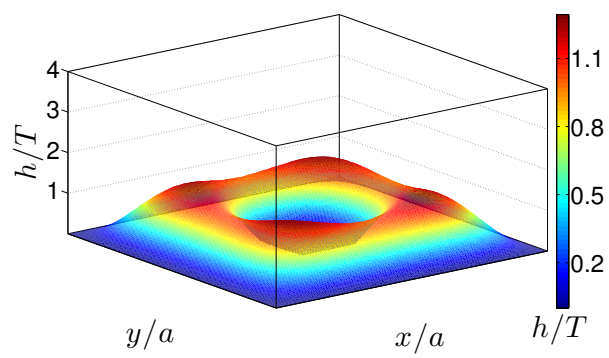

(c)

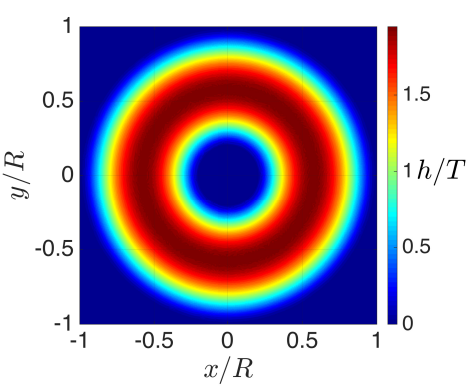

(b)

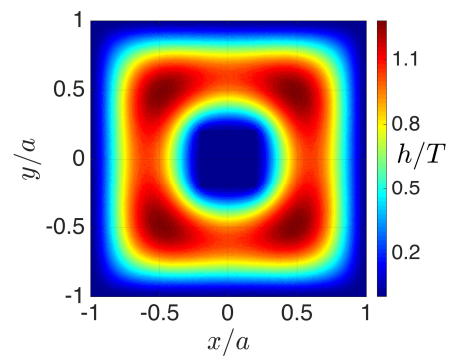

(d)

Figure 10: Elevated and plan-view contour plots of the deformation of a doubly-connected circular silicone pocket of radius $R=10 \mathrm{~mm}$ and $\alpha=0.2$ for sheet sample 2 at a dimensionless pressure $P R^{4} / E T^{4}=580(\mathrm{a}-\mathrm{b})$ and a doubly-connected square silicone pocket with $a=20 \mathrm{~mm}$ and $\alpha=0.2$ for sheet sample 3 at a dimensionless pressure $P a^{4} / E T^{4}=138$ (c-d), both obtained numerically using a finite element simulation. 


\subsubsection{Profiles}

The profiles of analytical and numerical normalised deflection for doublyconnected circular and square shapes for $\alpha=0.2$ are shown for $P R^{4} / E T^{4}$ and $\mathrm{Pa}^{4} / E T^{4}$ ranging from 0.8 to $8 \times 10^{4}$ in figure 11 . The eight numerical results (four for the doubly-connected circle and four for the doubly-connected square) are obtained for a pocket thickness, length, material specific constants and applied pressure range similar to section 4.2.3. In figure 11 (a), (10) matches perfectly the simulation of a doubly-connected circular shape for $\alpha=0.2$ and $P R^{4} / E T^{4}=0.8$. As $P R^{4} / E T^{4}$ and $P a^{4} / E T^{4}$ increase to 800, 8000 and 80000, it can be seen that the profiles in figure 11 (a) and (b) are more curved, in a similar fashion to figure 8 denoting the stretching regime.

\subsubsection{Maximum displacements}

In figure 12 , the dimensionless maximum deflections, $H / T$, for doubly-connected circular and square shapes are shown for a range of non-dimensional pressure $P R^{4} / E T^{4}$ and $P a^{4} / E T^{4}$ ranging from $8 \times 10^{-3}$ to $6.2 \times 10^{4}$ and $\alpha=0.1$, 0.2, 0.4 and 0.8. The forty-eight simulations (twenty-four for the doublyconnected circle and twenty-four for the doubly-connected square) were achieved with similar numerical parameters to section 4.3.2. Akin figure $9, H / T \leq 1$ corresponds to the bending regime while $H / T>1$ corresponds to the stretching regime. For the bending regime of a doubly-connected circular shape, the maximum non-dimensional height, $H / T$, deducted from the analytical solution (10) matches the numerical curves for all values of $\alpha$. It can be calculated that, for identical values of $P R^{4} / E T^{4}, H / T$ is reduced by more than a factor 


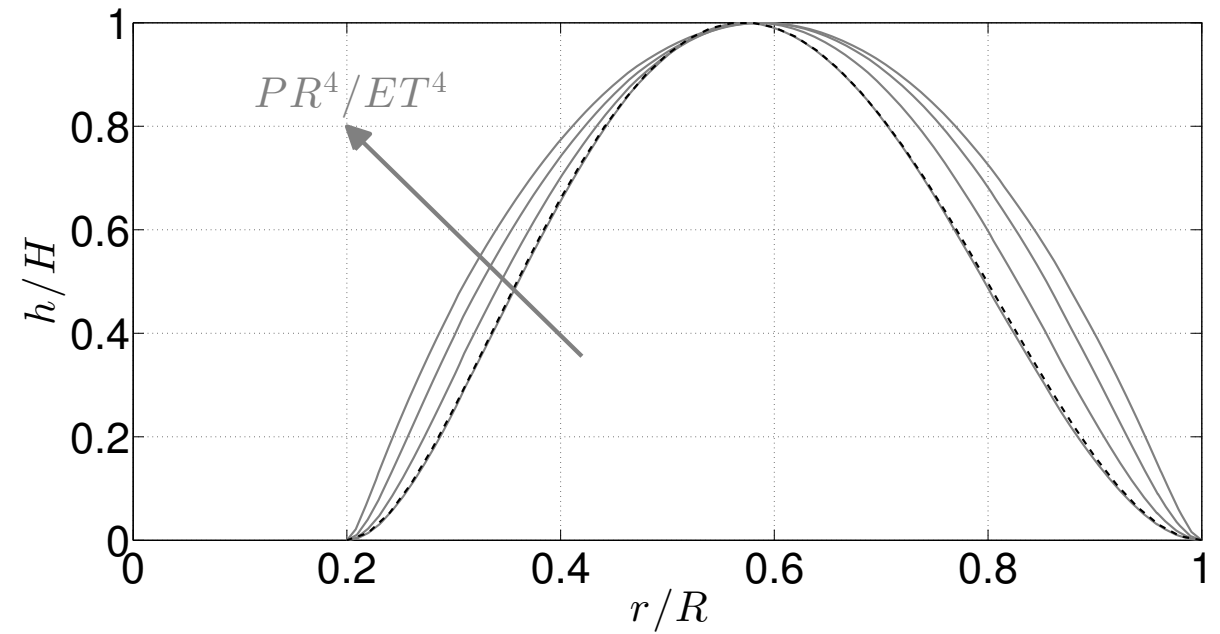

(a)

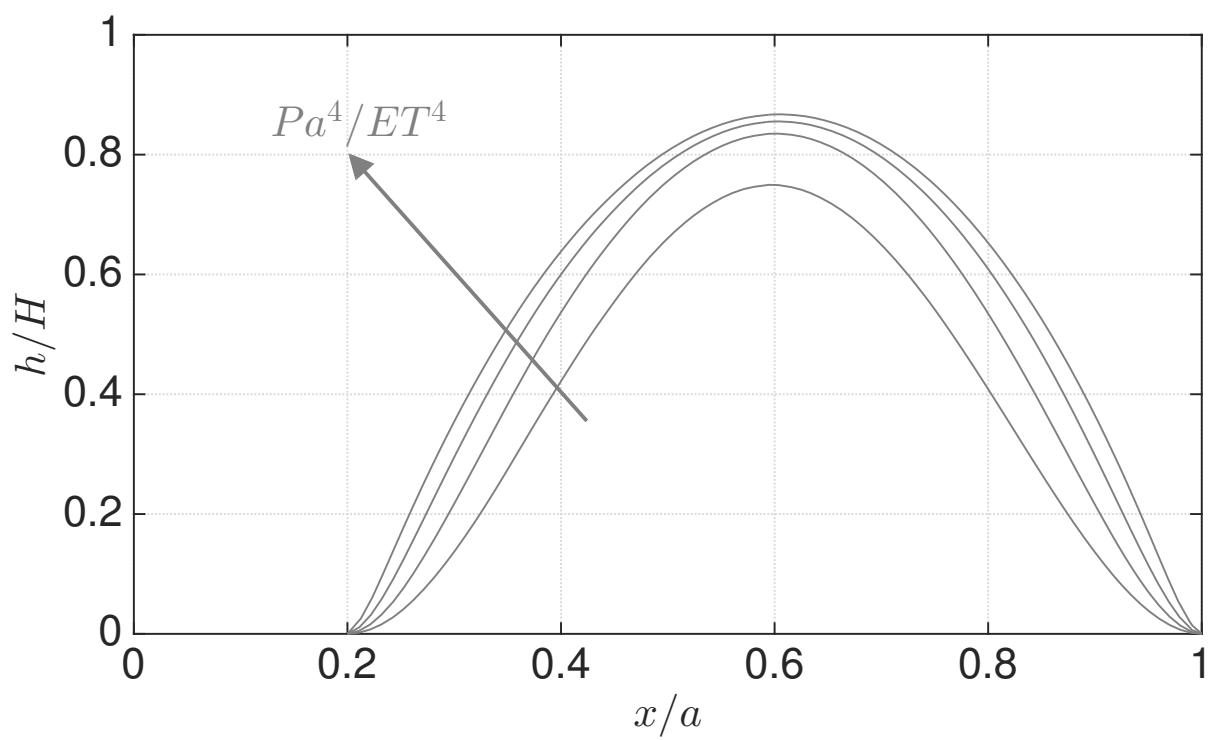

(b)

Figure 11: Profile of the sheet deflection along a line of symmetry for a doubly-connected (a) circular and (b) square silicone pocket with $\alpha=0.2$, as a function of dimensionless pressure $P R^{4} / E T^{4}$ and $P a^{4} / E T^{4}$ ranging from 0.8 to $8 \times 10^{4}$ obtained analytically (--), and numerically using a finite element simulation $(-)$. 
9 when considering a doubly-connected circular shape with $\alpha=0.1$ versus a simply-connected circular shape with similar geometric and material parameters. This reduction in height for a fixed dimensionless pressure is further amplified when $\alpha$ increases in both the doubly-connected circle and square cases. Therefore, the characteristic length $R$ and $a$ can be amended to $R(1-$ $\alpha$ ) for a doubly-connected circle and $a(1-\alpha)$ for a doubly-connected square to reflect the great dependence on $\alpha$. The dimensionless maximum deflection height, $H / T$, can then be rewritten in the case of a doubly-connected circle to:

$$
H / T=\gamma_{b}(1-\alpha)^{4}\left(\frac{P R^{4}}{E T^{4}}\right),
$$

with $R=a$ for a doubly-connected square. In the case of a ring, when $\alpha$ tends to 1, Hewitt et al. (2015) obtained an asymptotical approximation of $\gamma_{b}$ equal to $\left(1-\sigma^{2}\right) / 32$. In the stretching regime, the maximum deflection reduces as well when the size of the hole $(\alpha)$ increases for both shapes. For example, a doubly-connected circular disk with $\alpha=0.8$ and $P R^{4} / E T^{4}=$ $10^{4}$ bends $(H<T)$, while for a simply-connected circular disk, it stretches $(H>10 \times T)$. This shows the great dependence of $H$ and thus $h(r)$ on $\alpha$. Similarly to (19), $H / T$ can also be defined in the stretching regime for a doubly-connected circle as:

$$
H / T=\gamma_{s}(1-\alpha)^{4 / 3}\left(\frac{P R^{4}}{E T^{4}}\right)^{1 / 3},
$$

with $R=a$ for a doubly-connected square. In the case of a ring, $\gamma_{s}$ can be rewritten as approximately $\left(3\left(1-\sigma^{2}\right)\right)^{1 / 3} / 4$ when $\alpha$ tends to 1 (Hewitt et al., 2015). Details of $\gamma_{s}$ and $\gamma_{b}$ for both doubly-connected shapes are given in figure 13 . 


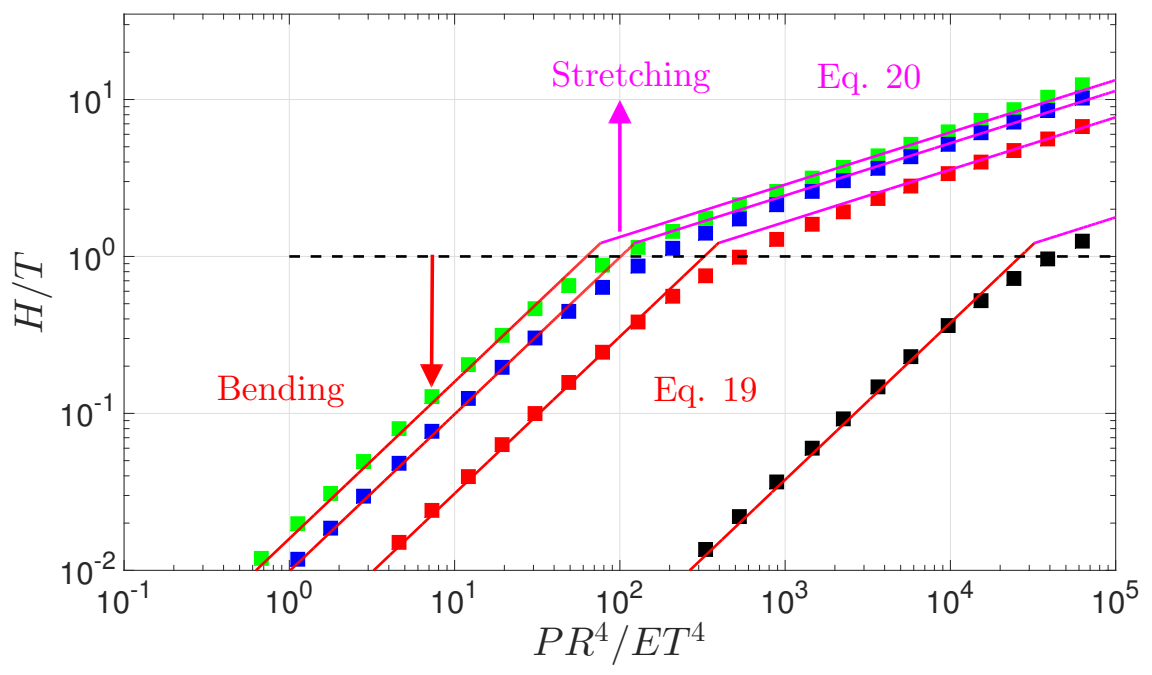

(a)

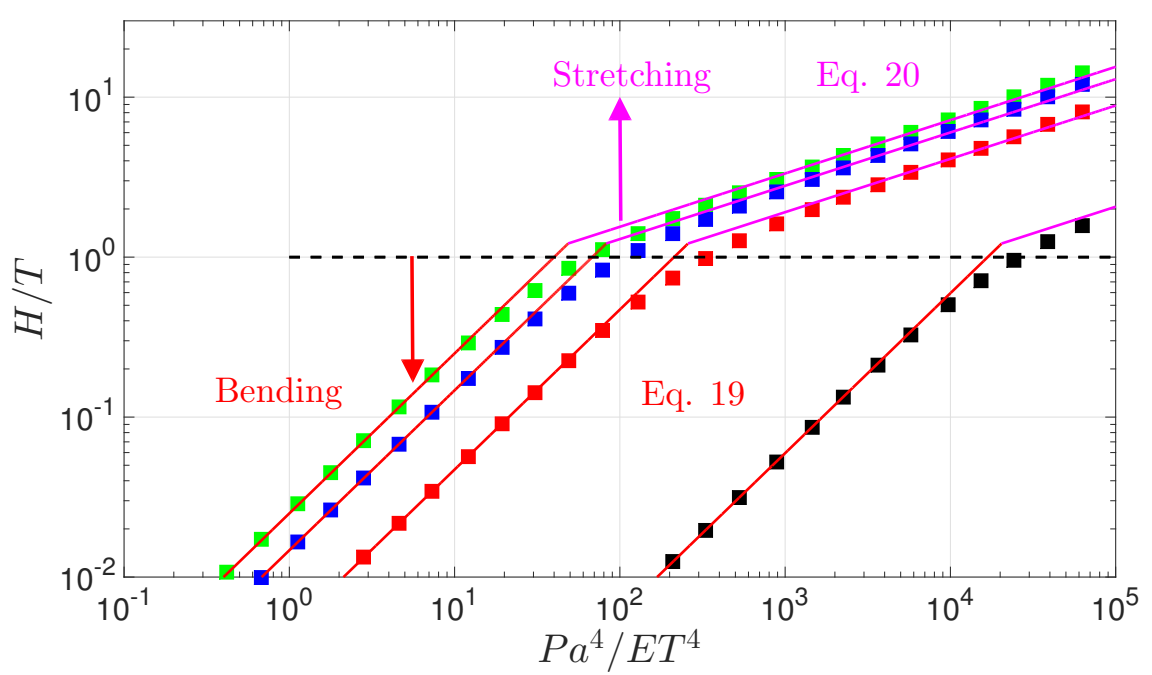

(b)

Figure 12: Variation of the dimensionless maximum deflection $(H / T)$ with the dimensionless pressure $\left(P R^{4} / E T^{4}\right)$ and $\left(P a^{4} / E T^{4}\right)$ for two different doubly-connected shapes in the case of circles (a) and squares (b) obtained numerically using finite element for $\alpha=0.1$ $(\square), \alpha=0.2(\square), \alpha=0.4(\square)$ and $\alpha=0.8(\mathbf{\square})$. The red curves are the analytical (a) and numerical (b) results for the bending regime and the magenta curves are obtained numerically for the stretching regime. 


\subsection{Comparison between simply and doubly-connected pockets}

A qualitative comparison based on maximum deflection is conducted between simply and doubly-connected pockets. The plots of the bending coefficients $\gamma_{b}$ and stretching coefficients $\gamma_{s}$ in figure 13 are based on thirtysix simulations (eighteen for the doubly-connected circle and eighteen for the doubly-connected square) with $\alpha$ ranging from 0.1 to 0.9 , and nondimensional pressure increasing up to $1.21 \times 10^{6}$ with other numerical parameters such as pocket thickness, length and material specific constants similar to section 4.3.2. Asymptotical solutions obtained when $\alpha$ tends to 1 from Hewitt et al. (2015) are reported with a dashed black line for the bending solution $\left(\left(1-\sigma^{2}\right) / 32\right)$ and a blue dashed line for the stretching solution $\left(\left(3\left(1-\sigma^{2}\right)\right)^{1 / 3} / 4\right)$. The analytical constant coefficients for simply-connected pockets obtained from $(9)\left(3\left(1-\sigma^{2}\right) / 16\right)$ in the case of a circle and (14) $\left(0.245\left(1-\sigma^{2}\right)\right)$ in the case of a square are also reported with respectively red dashed and dotted lines. These coefficients are the equivalents of $\gamma_{b}$ for simply-connected shapes. Similarly, magenta dashed and dotted lines represent respectively the stretching constant coefficients for simply-connected circles and squares obtained in (18). The coefficient $\gamma$ is the equivalent of $\gamma_{s}$ for simply-connected shapes and is equal to 0.6 for circle and 0.7 for square.

First, it can be noticed that both values of $\gamma_{b}$ for the doubly-connected shapes have a variation of a few percents over the range $\alpha=0.1-0.9$, for example in the case of the circle, $\gamma_{b}$ decreases from $24.09 \times 10^{-3}$ at $\alpha=0.1$ to $23.44 \times 10^{-3}$ at $\alpha=0.9$ converging towards the asymptotic approximation when $\alpha$ tends to 1 given by Hewitt et al. (2015) plotted with a black 
dashed line. Similarly, when considering stretching, $\gamma_{s}$ decreases slowly with a variation of respectively $6 \%$ and $11 \%$ for doubly-connected square and circle over the range $\alpha=0.1-0.9$. The asymptotic stretching approximation reported by Hewitt et al. (2015) for doubly-connected circle are within 1\% of the numerical simulations.

Moreover, $\gamma_{b}$ and $\gamma_{s}$ for both doubly-connected shapes are consistently lower than their equivalent parts for simply-connected shapes by approximately on average one order of magnitude for bending and slightly more than $40 \%$ in the case of stretching.

Finally, similarly to simply-connected shapes, the coefficients $\gamma_{b}$ and $\gamma_{s}$ are always higher in the case of doubly-connected squares compared to doublyconnected circles by an average of $50 \%$ in the case of bending and an average of $20 \%$ in the case of stretching. 


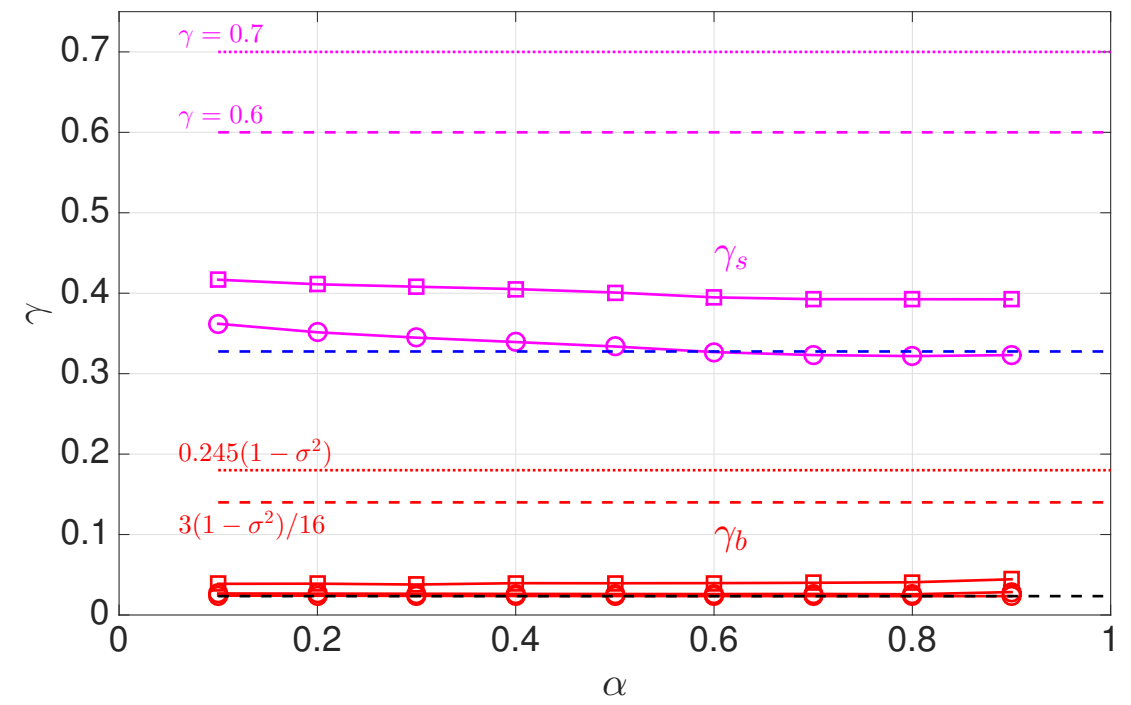

Figure 13: Bending (red) and stretching (magenta) coefficients $\left(\gamma_{b}\right.$ and $\left.\gamma_{s}\right)$ obtained from analytical and numerical analysis in the case of doubly-connected circles $(-\circ)$ and squares $(-\square)$ for increasing clamped central circular and square area ratio $(\alpha)$. Asymptotical bending and stretching solutions for a round annulus from Hewitt et al. (2015) are shown respectively in dashed black and blue lines. The equivalent bending and stretching coefficients $(\gamma)$ for simply-connected circles (dashed lines) and squares (dotted lines) are plotted respectively with red and magenta colours.

\section{Conclusions}

Pursing of simply and doubly-connected (square and circle) rubber planar elastic pockets have been quantified using analytical, numerical and experimental methods. When deflections are smaller than the thickness of the pocket, the pocket is bent and analytical solutions developed in previous works were sought (Koialovich, 1902; Boobnoff, 1902; Amon and Widera, 1969; Chandrashekhara, 2001; Meleshko, 1997; Hewitt et al., 2015), while 
when the deformations are greater than the thickness, the pocket is stretched and numerical solutions were extended using Finite Element Analysis.

The study was conducted to draw differences between pursed regime (bending and stretching) of different shapes (simply and doubly-connected) in terms of maximum deflections and profiles of pockets. The transition from bending to stretching has been studied in great detail. It was shown that the maximum deflection varies linearly with $P$ in the bending regime but to the power $1 / 3$ in the stretching regime once the maximum deflection reaches approximately the thickness of the pocket and continues to increase.

Both analytical and numerical approaches validated the optical methods to obtain the dimensionless maximum deflection $(H / T)$ of pursed silicone pockets for a range of dimensionless pressure $\frac{P R^{4}}{E T^{4}}$ from $10^{-1}$ to $10^{5}$. The profile widths of bent pockets compared to stretched pockets were also shown to be narrower as expected and quantified at different dimensionless pressures. It is worth nothing that the optical method was applied successfully for the first time to the pursing of different rubber pockets, if submitted to relatively low deflection. This technique can be applied to any sheet provided the material is clear.

A novel comparison was conducted between pursing of simply- and doublyconnected square and circle shapes. It was shown that the maximum deflection of doubly-connected pockets is greatly reduced compared to simplyconnected shapes with similar outer shapes. This difference was quantified by calculating and comparing the coefficients $\gamma_{b}$ and $\gamma_{s}$ to their equivalents for simply-connected shapes. For example, in the bending regime, the maximum deflection can be reduced by 5 orders of magnitude when the clamped 
central circular area ratio is $90 \%(\alpha=0.9)$ compared to a simply-connected

circular pocket for identical dimensionless pressure $\frac{P R^{4}}{E T^{4}}$. Therefore, a doublyconnected shape and more generally a multiply-connected shape has a significant effect on regime and response.

The authors believe that simply- and multiply-connected pursed elastic pockets could be used as effective drug reservoirs for continuous delivery.

Acknowledgements The authors acknowledge the support of the UK National Institute for Health Research Biomedical Research Centre at Moorfields Eye Hospital and the UCL Institute of Ophthalmology, the Helen Hamlyn Trust in memory of Paul Hamlyn, and Moorfields Eye Charity. This research has received a portion of its funding from the UK National Institute for Health Research. The authors would also like to thank Professor Steve Brocchini for the support of the research conducted on pursed planar elastic pockets, Lady Peggy Khaw, Research Programme Manager for all the daily support for the research and Dr. Paul Fromme for providing technical advice on Abaqus.

\section{References}

Adkins, J. E., Rivlin, R. S., 1952. Large elastic deformations of isotropic material IX. Philos. Trans. R. Soc. Lond. Ser. A 244, 505-531.

Amon, R., Widera, O. E., 1969. Clamped annular plate under a concentrated force. AIAA J. 7 (1), 151-153.

Boobnoff, I. G., 1902. Stresses in ship plates under water pressure. Morsk. Sb. 312 (10), 119-138. 
Bylski, D. I., Kriewall, T. J., Akkas, N., Melvin, J. W., 1986. Mechanical behavior of fetal dura mater under large deformation biaxial tension. J. Biomech. 19 (1), 19-26.

Cenedese, C., Dalziel, S., 1998. Concentration and depth field determined by the light transmitted through a dyed solution. 8th International Symposium on Flow Visualization, 61.1-61.5.

Chandrashekhara, K., 2001. Theory of Plates. Universities Press.

Chaplain, M. A. J., Sleeman, B. D., 1990. An application of membrane theory to tip morphogenesis in acetabularia. J. Theor. Biol. 146, 177-200.

Chaudhuri, A., DasGupta, A., 2014. On the static and dynamic analysis of inflated hyperelastic circular membranes. J. Mech. Phys. Solids 64, 302315.

Chien, W. Z., 1948. Asymptotic behavior of a thin clamped circular plate under uniform normal pressure at very large deflection. Sci. Rep. Natl. Tsing Hua Univ. 5, 71-86.

Christensen, R. M., Feng, W. W., 1986. Nonlinear analysis of the inflation of an initially flat, circular, elastic disk. J. Rheol. 30, 157-165.

Davies, G. C., Hiller, S., Bruce, D. M., 1998. A membrane model for elastic deflection of individual plant cell walls. J. Texture Stud. 29, 645-667.

Dickey, R. W., 1967. The plane circular elastic surface under normal pressure. Arch. Ration. Mech. Anal. 26, 219-236. 
Feng, W. W., 1976. Large plane deformations of rectangular elastic sheets. J. Appl. Math. Phys. (ZAMP) 27, 781-789.

Gent, A. N., Lewandowski, L. H., 1987. Blow-off pressures for adhering layers. J. Appl. Polym. Sci. 33, 1567-1577.

Glozman, D., Hassidov, N., Senesh, M., Shoham, M., 2010. A self-propelled inflatable earthworm-like endoscope actuated by single supply line. IEEE Trans. Biomed. Eng. 57 (6), 1264-1272.

Goulbourne, N. C., Frecker, M. I., Mockensturm, E. M., 2004. Electro-elastic modeling of a dielectric elastomer diaphragm for a prosthetic blood pump. Proc. SPIE 5385 5385, 122-133.

Goulbourne, N. C., Mockensturm, E. M., Frecker, M. I., 2007. Electroelastomers: large deformation analysis of silicone membranes. Int. J. Solids Struct. 44 (9), 2609-2626.

Hached, S., Loutochin, O., Corcos, J., 2014. Novel, remotely controlled, artificial urinary sphincter: a retro-compatible device. IEEE/ASME Trans. Mechatron. 19 (4), 1352-1362.

Hart-Smith, L. J., Crisp, J. D. C., 1967. Large elastic deformations of thin rubber membranes. Int. J. Eng. Sci. 5, 1-24.

Hencky, H., 1915. Uber den spannungszustand in kreisrunden platten. Z. für Math. und Phys. 63, 311-317.

Hewitt, I. J., Balmforth, N. J., De Bruyn, J. R., 2015. Elastic-plated gravity currents. Eur. J. Appl. Math. 26, 1-31. 
Imrak, C. E., Gerdemeli, I., 2007a. An exact solution for the deflection of a clamped rectangular plate under uniform load. Appl. Math. Sci. 1 (43), 2129-2137.

Imrak, C. E., Gerdemeli, I., 2007b. The problem of isotropic rectangular plate with four clamped edges. Sādhanā 32 (3), 181-186.

Jenkins, C. H., Leonard, J. W., 2015. Nonlinear dynamic response of membranes: State of the art. Appl. Mech. Rev. 44 (7), 319-328.

Jones, R., 1974. A simplified approach to the large deflection of membranes. Int. J. Non-Linear Mech. 9, 141-145.

Kao, R., Perrone, N., 1971. Large deflections of axisymmetric circular membranes. Int. J. Solids Struct. 7, 1601-1612.

Karimi, A., Navidbakhsh, M., Shojaei, A., Hassani, K., Faghihi, S., 2014. Study of plaque vulnerability in coronary artery using Mooney-Rivlin model: a combination of finite element and experimental method. Biomed. Eng.: Appl. Basis Commun. 26 (1), 1450013.

Kelkar, A., Elber, W., Raju, I. S., 1985. Large deflections of circular isotropic membranes subjected to arbitrary axisymmetric loading. Comput. Struct. 21 (3), 413-421.

Klingbeil, W. W., Shield, R. T., 1964. Some numerical investigations on empirical strain-energy functions in the large axisymmetric extensions of rubber membranes. Z. für Angew. Math. und Phys. ZAMP 15, 608-629. 
Koialovich, B. M., 1902. On One Partial Differential Equation of the Fourth Order (Doctor dissertation). St. Petersburg University Press, St. Petersburg.

Kriewall, T. J., Akkas, N., Bylski, D. I., Melvin, J. W., Work, B. A. J., 1983. Mechanical behavior of fetal dura mater under large axisymmetric inflation. J. Biomech. Eng. 105, 71-76.

Kumar, N., DasGupta, A., 2013. On the contact problem of an inflated spherical hyperelastic membrane. Int. J. Non-Linear Mech. 57, 130-139.

Landau, L. D., Lifshitz, E. M., 1975. Theory of Elasticity 3rd edition. Pergamon Press.

Levy, S., 1942. Report no. 740 - square plate with clamped edges under normal pressure producing large deflections. Natl. Advis. Comm. Aeronaut. $740,209-222$.

Liang, D. K., Yang, D. Z., Qi, M., Wang, W. Q., 2005. Finite element analysis of the implantation of a balloon-expandable stent in a stenosed artery. Int. J. Cardiol. 104 (3), 314-318.

Lister, J. R., G., P. G., Neufeld, J. A., 2013. Viscous control of peeling an elastic sheet by bending and pulling. Phys. Rev. Lett. 111, 154501.

Marker, D. K., Jenkins, C. H., 1997. Surface precision of optical membranes with curvature. Opt. Express 1 (11), 324-331.

Martin, D., Boyle, F., 2013. Finite element analysis of balloon-expandable 
coronary stent deployment: influence of angioplasty balloon configuration. Int. J. Numer. Methods Biomed. Eng. 29 (11), 1161-1175.

Meleshko, V. V., 1997. Bending of an elastic rectangular clamped plate: exact versus 'engineering' solutions. J. Elast. 48, 1-50.

Mooney, M., 1940. A theory of large deformation. J. Appl. Phys. 11, 582-592.

Morino, M., Toppino, M., Bonnet, G., Del Genio, G., 2003. Laparoscopic adjustable silicone gastric banding versus vertical banded gastroplasty in morbidly obese patients. Ann. Surg. 238 (6), 835-842.

Patil, A., DasGupta, A., 2013. Finite inflation of an initially stretched hyperelastic circular membrane. Eur. J. Mech. - A/Solids 41, 28-36.

Pihler-Puzović, D., Juel, A., Peng, G. G., Lister, J. R., Heil, M., 2015. Displacement flows under elastic membranes. part 1. experiments and direct numerical simulations. J. Fluid Mech. 784, 487-511.

Pistriakoff, D., 1910. La flexion d'une plaque mince. Izv. Kiev. Politekh. Inst. 10, 311-373.

Prendergast, P. J., 2003. Analysis of prolapse in cardiovascular stents: a constitutive equation for vascular tissue and finite-element modelling. J. Biomech. Eng. 125 (5), 692.

Pujara, P., Lardner, T. J., 1978. Deformations of elastic membranes-effect of different constitutive relations. Z. für Angew. Math. und Phys. ZAMP 29 (2), 315-327. 
Roberts, D. H., Green, W. A., 1980. Large axisymmetric deformation of a non-linear viscoelastic circular membrane. Acta Mech. 36, 31-42.

Selby, J. C., Shannon, M. A., 2009. Inflation of a circular elastomeric membrane into a horizontally semi-infinite liquid reservoir of finite vertical depth: Quasi-static deformation model. Int. J. Eng. Sci. 47, 700-717.

Smith, A. E., Moxham, K. E., Middelberg, A. P. J., 1998. On uniquely determining cell-wall material properties with the compression experiment. Chem. Eng. Sci. 53 (23), 3913-3922.

Song, W., Vasdekis, A. E., Psaltis, D., 2012. Elastomer based tunable optofluidic devices. Lab on a Chip 12 (19), 3590-3597.

Timoshenko, S. P., 1910. Sur l'application de la méthode des coordonnées normales au calcul de le flexion des tiges et des plaques. Izv. Kiev. Politekh. Inst. 10, 1-49.

Vossoughi, J., 1995. Determination of Mooney material constants for highly nonlinear isotropic incompressible materials under large elastic deformations. Exp. Tech. 19 (2), 24-27.

Weinitschke, H. J., 1988. On uniqueness of axisymmetric deformations of elastic plates and shells. SIAM J. Math. Anal. 19 (3), 580-592.

Wineman, A., 1978. On axisymmetric deformations of nonlinear viscoelastic membranes. J. Non-Newton. Fluid Mech. 4, 249-260.

Wineman, A., Wilson, D., Melvin, J. W., 1979. Material identification of soft tissue using membrane inflation. J. Biomech. 12, 841-850. 
Xu, S., Ren, H., Wu, S.-T., 2013. Dielectrophoretically tunable optofluidic devices. J. Phys. D: Appl. Phys. 46 (48), 483001.

Yang, W. H., Feng, W. W., 1970. On axisymmetrical deformations of nonlinear membranes. J. Appl. Mech. 37, 1002-1011. 